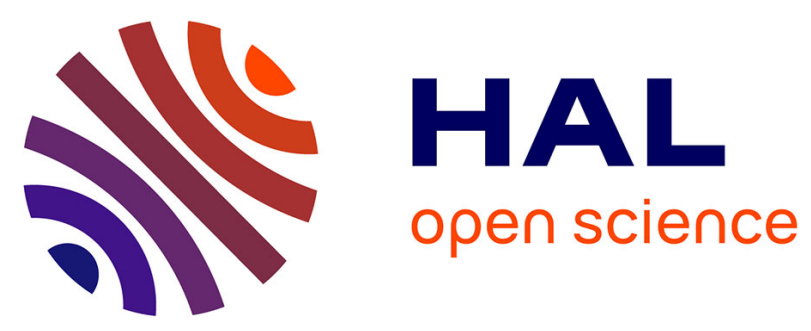

\title{
A complete system to determine the speed limit by fusing a GIS and a camera
}

\author{
Anne-Sophie Puthon, Fawzi Nashashibi, Benazouz Bradai
}

\section{To cite this version:}

Anne-Sophie Puthon, Fawzi Nashashibi, Benazouz Bradai. A complete system to determine the speed limit by fusing a GIS and a camera. ITSC'11 - 14th International IEEE Conference on Intelligent Transportation Systems, Oct 2011, Washington DC, United States. pp.1686-1691, 10.1109/ITSC.2011.6082951 . hal-00646592

\section{HAL Id: hal-00646592 \\ https://hal.science/hal-00646592}

Submitted on 30 Nov 2011

HAL is a multi-disciplinary open access archive for the deposit and dissemination of scientific research documents, whether they are published or not. The documents may come from teaching and research institutions in France or abroad, or from public or private research centers.
L'archive ouverte pluridisciplinaire HAL, est destinée au dépôt et à la diffusion de documents scientifiques de niveau recherche, publiés ou non, émanant des établissements d'enseignement et de recherche français ou étrangers, des laboratoires publics ou privés. 


\title{
A complete system to determine the speed limit by fusing a GIS and a camera
}

\author{
Anne-Sophie Puthon, Fawzi Nashashibi and Benazouz Bradai
}

\begin{abstract}
Determining the speed limit on road is a complex task based on the Highway Code and the detection of temporary speed limits. In our system, these two aspects are managed by a GIS (Geographical Information System) and a camera respectively. The vision-based system aims at detecting the roadsigns as well as the subsigns and the lane markings to filter those applicable. The two sources of information are finally fused by using the Belief Theory to select the correct speed limit. The performance of a navigation-based system is increased by $19 \%$.
\end{abstract}

\section{INTRODUCTION}

There were 100 millions of vehicles in 1986, 800 millions in 2005 and over 1 billion in 2010 [1] worldwide. The increase of individual transport seems to be unstoppable. In industrial countries, every household owns at least one or two cars. However, great improvements have been achieved, particularly in driver assistance systems, between the past and the current models. The objectives are to make the car comfortable, safe and environmentally friendly. Intelligent Speed Adaptation (ISA) systems are directly related to these issues. What are the consequences if the car is indeed able to determine the current speed limit and eventually adapt its speed to this value? First, this represents a safety improvement because speed remains one of the most lethal causes on roads. Second, a comfort enhancement is expected as speed adaptation becomes an automated process. Third, an obvious fuel consumption reduction is predictable due to these systems [2].

Existing commercial products focus on the detection of speed signs on the road side with a camera. Some developed systems also implement Geographical Information Systems (GIS), like the LAVIA project [3]. Some approaches use both sensors. This kind of autonomous system, relying on embedded sensors, is less intrusive than cooperative ones. They do not require any modification in the infrastructure or any supplementary communication device. Furthermore, they appear to work like a Human, by analyzing the surrounding environment and extracting the relevant information.

Manuscript received July 31st, 2011.

A.-S. P. is from the Robotics Centre of Mines ParisTech, 60 boulevard Saint-Michel F-75272 Paris Cedex 06, FRANCE (phone: (33)1-40-51-9454, email: anne-sophie.puthon@ensmp.fr)

F. N. is both from the Robotics Centre of Mines ParisTech and from INRIA, IMARA Team, BP 105 F-78153 Le Chesnay Cedex, FRANCE (phone: (33)1-39-63-52-56, email: fawzi.nashashibi@ensmp.fr)

B. B. is from Valeo Driving Assistance Research Center, 34 rue Saint André F-93012 BOBIGNY Cedex, FRANCE (phone: (33)1-49-42-60-95, email: benazouz.bradai@valeo.com)
Ideally, ISA systems would be able to predict the effective speed limit at any time. However, current applications do not perform this task completely. Camera-based approaches aim at detecting traffic signs, representing temporary limits, thus ignoring general rules specified by the Highway Code. On the contrary, GIS only gives prerecorded information relative to a given position. Their knowledge about the environment may be ouf-of-date. A fusion between these two complementary sources is thus recommended to obtain the lowest failure rate possible. It efficiently combines the dynamic vision multifunctionality with the global knowledge and availability of GIS.

Our article is structured as follows. First, our idea of an ideal ISA system based on the Human reasoning is depicted (Sec. II). Then, we describe how implicit speed limits are determined (Sec. III) and how a camera-based system detects and analyzes eventual traffic signs (Sec. IV). The fusion of these two sources of information is then performed thanks to a method based on the belief theory (Dempster-Shafer) and the final decision is made (Sec. V). Consequently, section VI presents the results comparing systems bringing into play different architectures. To finish, we conclude our work and introduce some perspectives about future implementations.

\section{TOWARD AN IDEAL ISA SYSTEM}

\section{A. From Human...}

Nowadays, more and more intelligent systems are developed in order to help or replace the Human in monotonous, dangerous or critical situations. Despite this laudable goal and incredible growth, these systems are still hardly accepted by users. In case of safety systems, like ISA, this mistrust is even more noticeable. In fact, the driver would be more inclined to follow advice if he understands the process of his assistant. The acceptance rate then depends on the similarity of the system's processing chain with the driver behavior. Studying and understanding the cognitive function of speed limit determination is thus the first step to succeed.

The Human reasoning is based on two sources of information: the Highway Code and the roadsign. The first one defines a set of general rules, or implicit speeds, valid under normal conditions and specific to certain driving situations. For example, figure 1 shows the limits applicable in France. Drivers deduce the driving context from their environment, for example the color of the guideposts, the road configuration or the presence of buildings. The second source of 


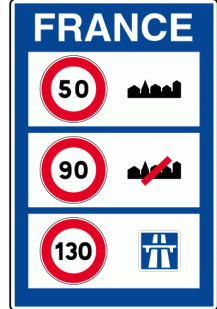

(a)

\begin{tabular}{|c|c|c|c|c|}
\hline \multicolumn{2}{|c|}{ Driving context } & \multicolumn{3}{c|}{ Speed limit (km/h) } \\
\hline Environment & Road type & Dry & Rain & Fog \\
\hline \multirow{3}{*}{ Non urban } & Highway & 130 & 110 & 50 \\
\cline { 2 - 5 } & Road with median trip & 110 & 100 & 50 \\
\cline { 2 - 5 } & Other road & 90 & 80 & 50 \\
\hline Urban & & 50 & 50 & 50 \\
\hline
\end{tabular}

(b)
Fig. 1. French implicit speeds. (a) Traffic sign located at the border to specify the general national rules concerning speed limits. (b) A more exhaustive list of the speed limits in France for cars, depending on weather conditions.

information, the roadsigns, gives temporary speed limits in some dangerous or unexpected situations, like roadworks or sharp bends. Sometimes restrictions are given as supplementary signs, specifying the vehicle category, the application area or time, etc. Implicit speed limits are therefore locally substituted by this new information until a new roadsign or the road exit appears.

\section{B. ... to Robot}

From the perspective of implementing an ISA system, easily acceptable and reliable, autonomous systems are more appropriate than cooperative ones. Embedded sensors act like the Human senses to analyze the environment and deduce the speed limit without requiring any change in the infrastructure or in the communication media with other systems. Thereby, the architecture reproduces the Human behavior as faithfully as possible. The global system consists of three main components (Fig. 2):

- a navigation-based system which aims at establishing the correspondence between a driving context and an implicit speed;

- a vision-based system to detect traffic signs, eventual supplementary signs and markings;

- a decisional part which fuses the information from both sources and outputs the most likely speed limit. In order to do that, it combines the influence of each sensor depending on its reliability and the previous result to avoid inconsistencies.

The configuration enables the system to detect any speed limit update: a driving context change (road change, city entrance, etc.) or a traffic sign.

\section{IMPLICIT SPEED LIMIT}

\section{A. Use of a navigation-based sensor}

Most of the time, effective speed limits only depend on the driving context. To acquire this knowledge, an ISA system must be able to extract and analyze information about the surrounding environment. This is made possible by the use of a navigation-based system. To achieve this goal, three successive steps: localization, map-matching and data extraction. First, a positioning tool, typically a Global Positioning System (GPS), locates the vehicle on Earth. Secondly, this information is combined with data

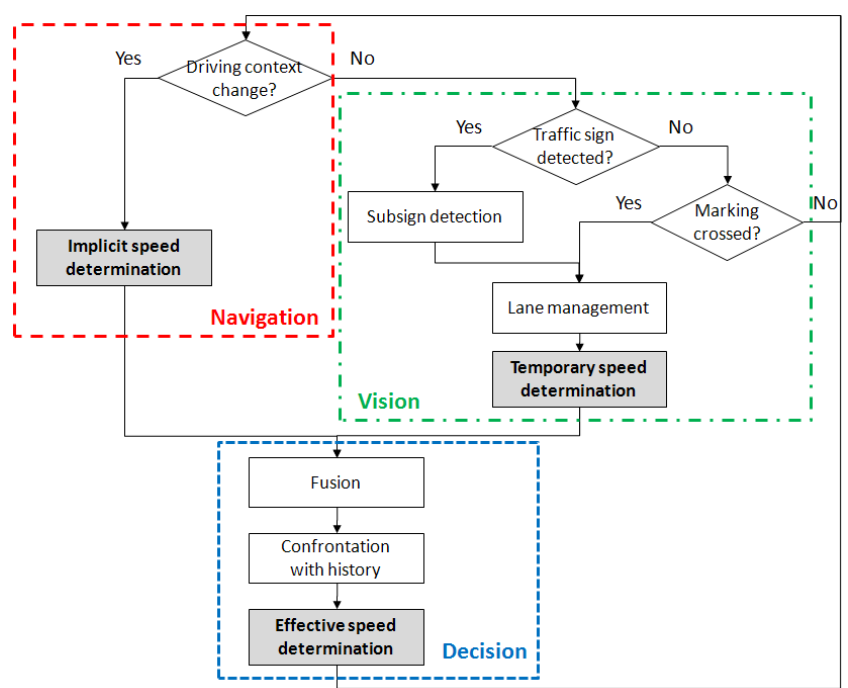

Fig. 2. Diagram of the complete system composed of three main parts: navigation- and vision-based systems and a decisional part. The speed limit update process is actuated by three types of event modeled by diamonds. Changes in the driving context are managed by the navigation. Newly detected traffic signs or marking crossings are both managed by the vision. The updated information from the two parts are then fused to determine the effective speed limit. The result is finally compared to the previous one to detect great gaps in speed revealing errors.

from Dead-Reckoning systems and odometers through map-matching algorithms. The most likely road of the network is thus selected [4]. Finally, previously stored information about the current road are extracted from the map database in order to analyze the driving context and determine the implicit speed limit.

Implementing such a tool has two main advantages: availability and simplicity. Indeed, using a GPS, which is more and more common in vehicles, avoids the installation of an additional sensor. Moreover, all the required data for determining the driving context are stored off-line, making them nearly immediately available. However, this system leads to inaccuracies. Standard GPS precision up to ten meters or signal loss due to the canyoning effect are typical sources of errors. An unfortunate consequence is the selection of a wrong road section, leading to wrong driving context and speed limit. Furthermore, the database can be out-of-date, e.g. in case of roadworks.

This relative imperfection leads us to prefer a statistical approach, computing a confidence in the correctness of each speed. In order to do that, criteria, are computed from the information extracted from the database. Each represents an aspect to consider in speed limit determination (Fig. 3).

\section{B. Extraction of criteria}

This concept was first introduced by [5] and [6] and developed by [7]. All the consistent information concerning the implicit speed limit determination is summarized by nine criteria, subdivided into two classes. The first, $C_{r e l}$, concerns the reliability of the sensor itself or the trust in the ability 


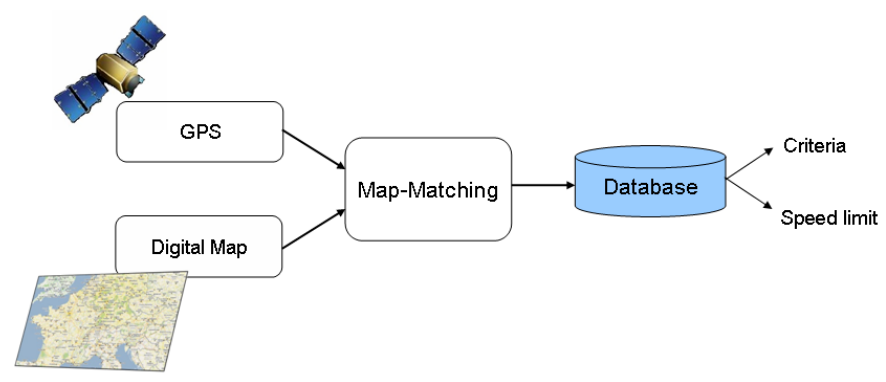

Fig. 3. Use of a navigation-based sensor. After having located the vehicle on the road network, attributes concerning the driving context are extracted from the database in order to determine the corresponding speed limit.

of the system to select the correct speed limit.

- $C_{r e l 1}$ : confidence in the positioning tool;

- $C_{r e l 2}$ : quality of the road digitization;

- $C_{r e l 3}$ : functional class, or the definition degree, of the road;

- $C_{\text {rel } 4}$ : guidance mode of the navigation system activated or not.

The second class, $C_{S L}$, helps in computing the confidence in each speed limit for a given driving context.

- $C_{S L 1}$ : type of the road (highway, primary and secondary roads, etc.);

- $C_{S L 2}$ : urban/non urban environment;

- $C_{S L 3}$ : crossing or not;

- $C_{S L 4}$ : highway exit or not;

- $C_{S L 5}$ : speed stored in the database or not. A higher confidence is assigned to a speed limit if it is specified in the database.

The confidences of both classes are then combined to compute a probability for the validity of each speed as illustrated in figure 4. More details are given in [7].

\section{TEMPORARY SPEED LIMIT}

\section{A. Traffic Sign Detection}

The role of roadsigns is to locally replace general rules. Their detection is thus critical for ADAS because, most of the time, they indicate the presence of a dangerous situation. The interest car manufacturers take in this task as well as the great amount of literature on the subject shows its importance. Commercial products are already available (e.g. MobilEye system in BMW7 Series [8]). Many techniques exist to achieve this goal, processing on either monochromatic or color images, like cross-correlation [9] or machine learning ([10], [11]).

Generally, three steps are necessary to determine such speed limits: detection, recognition and tracking of traffic signs. The method we used was developed by Bargeton et al. [11]. Circles are first detected in images with the Hough Transform method. The recognition step aims at separating real speed signs from the other circular shapes. Candidates are therefore binarized and segmented into digits, which are separately recognized by a neural network. A tracking finally

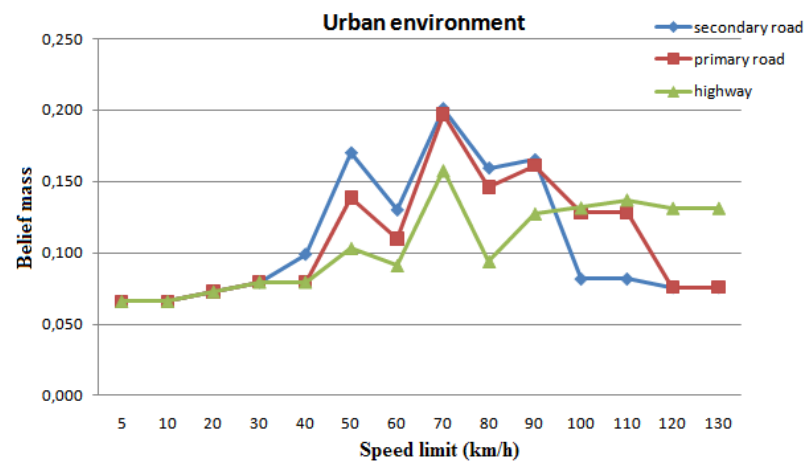

(a)

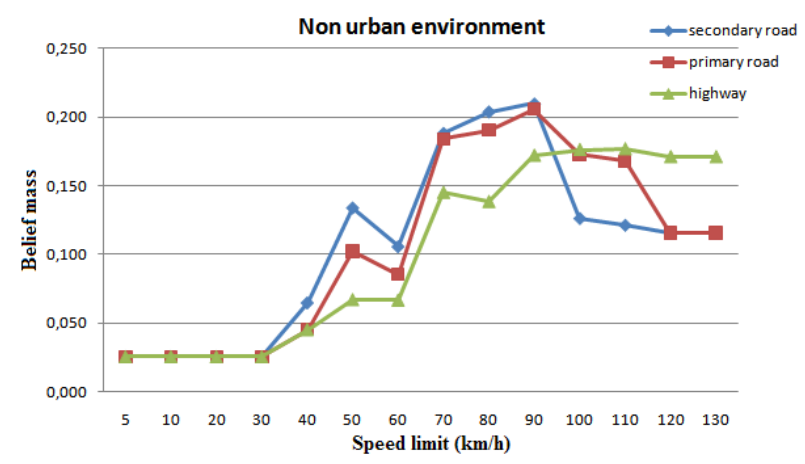

(b)

Fig. 4. Confidence in each speed limit in urban (a) and non urban (b) environments for three roadtypes: highway, primary and secondary road.

validates the speed limits. The final confidence in a given speed is related to the performance of each step.

\section{B. Subsign detection}

A validated traffic sign infers that the speed limit is applicable once the roadsign has been passed. However, some restrictions about the vehicle category or a given area can be specified. This additional information is given by rectangular signs, placed under the respective roadsign (Fig. 6). Fig. 5 shows some examples of subsigns frequently encountered on French roads. Although their detection seems essential to implement a complete system, conducted research is not currently well developed. Hamdoun et al. [12] proposed a method for detecting arrows, signalling the proximity of a highway exit, and some similarities exist with the detection of U.S. signs [13] or text on roadsigns [14].

As one of the most frequent situations with subsigns is highway exit, we implement the arrow detection of [12]. Based on the assumption that subsigns are located under their corresponding roadsign, we focus on searching for rectangles in this area, with an edge-based detector. Each of the candidates is then normalized and classified with a neural network. 


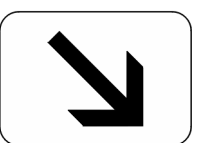

(a)

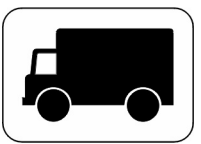

(b)

\section{$\uparrow 4,5 \mathrm{~km} \uparrow 500 \mathrm{~m}$}

(c)

(d)
Fig. 5. Some examples of French subsigns. (a) Effectiveness only on right lane. (b) Only trucks are concerned. (c) The subsign is effective for specified distance. (d) Subsign becomes effective at this distance from the current position.

\section{Lane management}

In some cases, a traffic sign is dedicated to a specific lane. This is the case of the highway exit for example. Lane management enables to assign detected speed signs to the right lanes and, in case of a lane crossing, to update a speed limit. Figure 6 illustrates a problematic case of a highway exit where lane management leads to the correct result. Two successive steps are implemented in our system. We first focus on detecting and classifying the markings on each side of the vehicle. The characterization is the main challenge in which our module excels. By evaluating the thickness, length and gap, our module is able to identify the marking type among the numerous existing patterns (Fig. 7). Secondly, an expert system combines information from the traffic sign and subsign detectors with the marking manager to assign the speed limit to the corresponding lane. More generally, the system studies the relative position of the roadsigns to the markings and lane crossing to determine the current road and the effective speed limit.

\section{DECISION}

\section{A. Principle}

After having collected data from the two sensors, the effective speed limit is determined through a decision process divided into three steps. Firstly, sensor data is combined. Using only a navigation-based system would require a perfectly up-to-date map database and a very accurate positioning tool, which seems impossible in practice. On the contrary, implicit speed limits are hardly computed with the help of a visionbased system. The fusion of both sensors appears then as the most efficient way of determining speed limits at any time. Secondly, irrelevant speed limits are filtered. In tricky situations or when sensors fail, inconsistencies appear which are eliminated by keeping in memory the previous speed limits. Finally, the decision is made by selecting the most likely candidate.

\section{B. Fusion with Dempster-Shafer}

Multisensor speed limit determination search is a topical issue ([15], [16], [6]). We valued the Dempster-Shafer over the Bayesian approach because of its good management of conflict and ignorance. The conflict occurs when sources give different speed limits and the ignorance when no information is available for one of the sensors. In addition, we implemented the discounting scheme of Mercier et al. [17] to take into account the reliability of the navigation

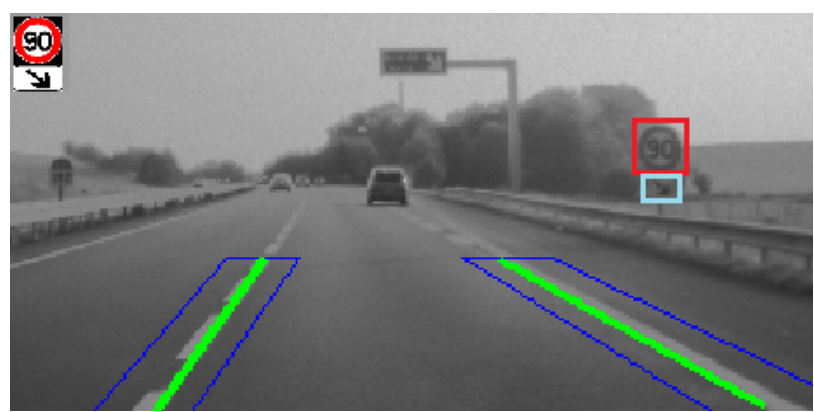

(a)

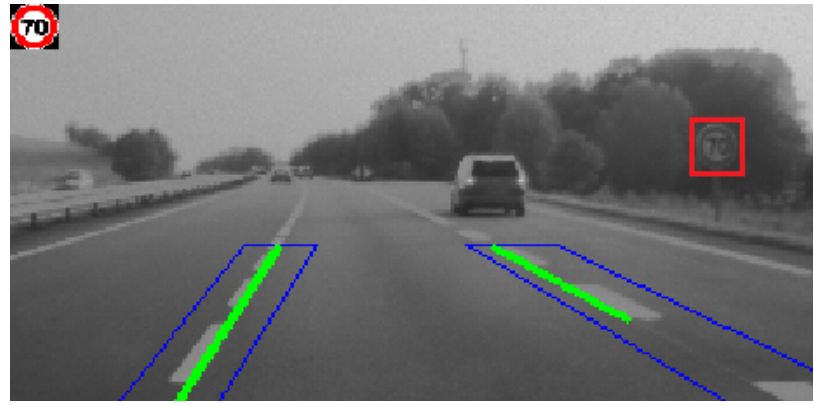

(b)

\begin{tabular}{|c|c|c|c|c|}
\hline & \multicolumn{3}{|c|}{ Speed limit } \\
\hline & & Before & After (a) & After (b) \\
\hline \multirow{3}{*}{ 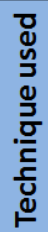 } & Traffic sign & 130 & 90 & 70 \\
\hline & $\begin{array}{l}\text { Traffic sign } \\
+ \text { Subsign }\end{array}$ & 130 & 130 & 70 \\
\hline & $\begin{array}{l}\text { Traffic sign } \\
\text { + Subsign } \\
\text { + Marking }\end{array}$ & 130 & 130 & 130 \\
\hline
\end{tabular}

(c)

Fig. 6. Highway exit situation management. The resulting speed limit is given in (c) with different configurations: traffic sign only, traffic sign and subsign and finally traffic sign, subsign and marking. In (b), with the lane management, all the traffic signs detected beyond the exit lane are discarded because the vehicle has not crossed the marking.

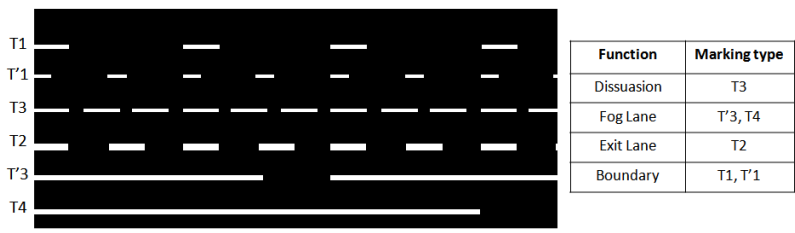

Fig. 7. French marking types and their significance.

sensor. Its influence is thus weighted by this performance factor.

We briefly introduce the concepts of Dempster-Shafer used in our application. Further details can be found in [18], [19] and [17]. The ground principle is based on belief mass $m$ representing the degree of belief in an event.

- the universe $D$ is the set of all $N$ possible speeds in France and the power set $\Omega$ is generated by all the 
subsets of $D$.

$$
\begin{aligned}
D= & \{5,10,15,20,30,45,50,60,70,80, \\
& 90,100,110,120,130\} \\
\Omega= & \{\emptyset,\{5\},\{10\},\{5,10\}, \ldots, D\}
\end{aligned}
$$

- the belief mass $m_{S}$ or the degree of belief in an event, given by a source $S$.

$$
m_{S}: \Omega \rightarrow[0,1], \quad \sum_{A \in \Omega} m_{S}(A)=1
$$

\section{- Discounting scheme}

$$
\left\{\begin{array}{l}
{ }^{\alpha} m_{S}(A)=(1-\alpha) m_{S}(A) \quad \forall A \subset D \\
{ }^{\alpha} m_{S}(D)=(1-\alpha) m_{S}(D)+\alpha
\end{array}\right.
$$

where ${ }^{\alpha} m_{S}$ is the belief mass using the discounting and $\alpha \in[0,1]$ a discounting rate. $(1-\alpha)$ represents the degree of belief in the source reliability.

- Conjunctive rule between two sources $S_{1}$ and $S_{2}$

$$
\left\{\begin{array}{l}
m(A)=\frac{1}{1-K} \sum_{B_{1} \cap B_{2}=A \neq \emptyset} m_{S_{1}}\left(B_{1}\right) m_{S_{2}}\left(B_{2}\right) \quad \text { if } A \neq \emptyset \\
m(\emptyset)=0
\end{array}\right.
$$

where $K=\sum_{B_{1} \cap B_{2}=\emptyset} m_{S_{1}}\left(B_{1}\right) m_{S_{2}}\left(B_{2}\right)<1$ is the normalization factor.

Two sources of information are available: the navigation $S_{\text {nav }}$ and the vision $S_{v i s}$. The fusion is then directly given in (4). How is each belief mass determined? For the visionbased system, $m_{S_{v i s}}$ is computed from the neural network output, which is a statistical value, and the tracking result. The belief mass of a given traffic sign increases proportionally to the number of successive frames in which it is recognized. Concerning the navigation-based system, the mass function comes from the discounting scheme. The reliability is estimated with the class $C_{r e l}$ and the belief in each speed related to the driving context with $C_{S L}$.

$$
\begin{aligned}
m_{r e l} & =\sum_{j=1}^{4} \alpha_{r e l} C_{r e l j} \\
m_{S L}(A) & =\sum_{k=1}^{5} \alpha_{S L k} C_{S L k}(A)
\end{aligned}
$$

$\alpha_{x}$ are weighting factors established empirically depending on the influence of each criteria [7].

$$
\left\{\begin{array}{l}
m_{S_{\text {nav }}}(A)=m_{\text {rel }} m_{S L}(A) \\
m_{S_{\text {nav }}}(D)=m_{\text {rel }} m_{S L}(D)+\left(1-m_{\text {rel }}\right)
\end{array} \quad \forall A \in D\right.
$$

\section{Final decision}

The best candidate is selected as the most likely speed limit in the sense of Dempster-Shafer. The decision rule consists thus in choosing the speed limit $d^{*}$ with the highest belief Bel.

$$
\begin{aligned}
\operatorname{Bel}(A) & =\sum_{B \subset A, B \neq \emptyset} m(B) \\
\operatorname{Bel}\left(d^{*}\right) & =\max _{1 \leq j \leq N} \operatorname{Bel}\left(d_{j}\right)
\end{aligned}
$$

At this moment, weather conditions have not been taken into account. However, the Highway Code specifies speed limits not only in function of the driving context but also of weather conditions (Fig. 1). If information from the CAN bus of the vehicle is available, like wipers or fog lamp state, this step is trivial. An alternative solution would be to implement image-based algorithms to estimate the weather conditions ([20], [21], [22]). The advantages are twofold: detecting precipitations and traffic signs with a single piece of equipment and quantifying the expected reliability of the camera.

\section{EVALUATION}

Our system is evaluated on four trajectories, one of them being acquired both on a sunny and a cloudy day (respectively $4 \mathrm{a}$ and $4 \mathrm{~b}$ ). Their characteristics are given in table I. The hardware architecture implemented consists of a 10-bit monochromatic camera with a resolution of $752 \times 480$ and a SensorBox Navteq. The data synchronisation is achieved with the RTMaps software, designed by Intempora (www.intempora.com) for real-time multisensor applications.

A ground truth corresponding to the effective speed limit is associated to each sequence. It takes into account the road type, the weather, the encountered traffic signs and if the vehicle is in an urban environment or not. To evaluate our system, we compare the outputs of the navigation and vision systems alone and their fusion with this ground truth. Table II summarizes the results obtained before and after the integration of the subsign detection and the lane management. Results correspond to the ratio of kilometers with the right speed limit.

The navigation system achieves good performance due to two factors: the up-to-date database on the road sections where the sequences were acquired and an accurate localisation. On the other hand, the use of the vision system alone is obviously not sufficient for our purpose. However, the subsign detection and the lane management modules improve the results by discarding some roadsigns indicating speed limits for road exits. Finally, the fusion of both sensors leads to better performance of the system but also to more robustness in case of a failure of one sensor. In case of a cloudy sky (table II - sequence $4 \mathrm{~b}$ ), the results of both navigation and vision drop to $27 \%$ and $17 \%$ respectively. Nevertheless, the global system performance achieves an accuracy of up to $87 \%$.

\section{CONCLUSION}

We present a complete system to determine effective speed limit on road with respect to the Human reasoning. Hence, we combine a navigation- and a vision-based system, leading to better performance. Subsign recognition and lane managing brought substantial improvements to the existing architectures by performing fusion between navigation and 


\begin{tabular}{|c|c|c|c|c|}
\hline \multirow{2}{*}{} & \multicolumn{4}{|c|}{ Sequence } \\
\cline { 2 - 5 } & $\mathbf{1}$ & $\mathbf{2}$ & $\mathbf{3}$ & $\mathbf{4}$ \\
\hline Duration & $0: 36: 13$ & $0: 35: 53$ & $1: 18: 43$ & $0: 51: 42$ \\
\hline Length & $64.7 \mathrm{~km}$ & $20.3 \mathrm{~km}$ & $124.1 \mathrm{~km}$ & $54.9 \mathrm{~km}$ \\
\hline City proportion & $1 \%$ & $86 \%$ & $11 \%$ & $11 \%$ \\
\hline Highway proportion & $99 \%$ & $99 \%$ & $88 \%$ & $0 \%$ \\
\hline Road exits & 11 & 11 & 27 & 20 \\
\hline Roadsigns & 58 & 43 & 106 & 68 \\
\hline Subsigns & 12 & 9 & 5 & 23 \\
\hline
\end{tabular}

TABLE I

SEQUENCES OF THE DATASET USED FOR THE EVALUATION.

\begin{tabular}{|c|c|c|c|c|c|}
\hline & & \multicolumn{2}{|c|}{ Before } & \multicolumn{2}{c|}{ After } \\
\cline { 3 - 6 } Sequence & Navigation & Vision & Fusion & Vision & Fusion \\
\hline 1 & $81 \%$ & $47 \%$ & $67 \%$ & $48 \%$ & $86 \%$ \\
\hline 2 & $98 \%$ & $74 \%$ & $90 \%$ & $93 \%$ & $98 \%$ \\
\hline 3 & $69 \%$ & $48 \%$ & $60 \%$ & $45 \%$ & $74 \%$ \\
\hline $4 \mathrm{a}$ & $65 \%$ & $45 \%$ & $56 \%$ & $69 \%$ & $84 \%$ \\
\hline $4 \mathrm{~b}$ & $27 \%$ & - & - & $17 \%$ & $87 \%$ \\
\hline
\end{tabular}

TABLE II

PERFormanCE OF THE DifFERENT SYSTEMS BEFORE AND AFTER THE INTEGRATION OF THE SUBSIGN DETECTION AND THE LANE

MANAGEMENT. RATIOS CORRESPOND TO THE AMOUNT OF KM WITH THE CORRECT SPEED LIMIT.

traffic sign recognition.

However, some issues still remain unresolved. As mentioned, a stand-alone ISA system needs to be nearly perfect under any condition. The most obvious problematic situation is when bad weather conditions disturb the vision-based algorithm (Fig. 8). We therefore plan to couple our vision module with a precipitation detector under development [22] to estimate the accuracy loss due to bad visibility conditions. Moreover, we work on a system able to detect and recognize any type of subsign in order to fully manage the traffic sign indications.

\section{ACKNOWLEDGMENTS}

This work was conducted in the framework of the Speedcam project (ANR-09-VTT-11), funded by the ANR (Agence Nationale de la Recherche).

\section{REFERENCES}

[1] "www.polk.com."

[2] S. Aupetit, "Approche Ergonomique de l'Usage du Régulateur de Vitesse Conventionnel: Analyse Critique de la Littérature Scientifique," Revue Électronique, p. 104, 2010.
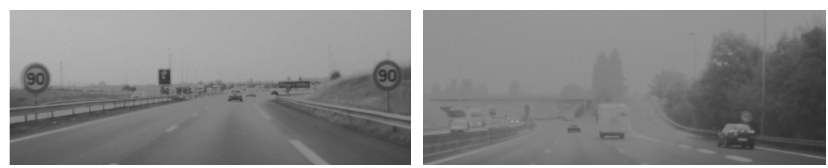

Fig. 8. Comparison of acquired images in normal (a) and in rainy (b) situations. As the presence of rain deteriorates the image quality, errors occur in the traffic sign recognition.
[3] R. Driscoll, Y. Page, S. Lassarre, and J. Ehrlich, "LAVIA-An Evaluation of the Potential Safety Benefits of the French Intelligent Speed Adaptation Project." Annual proceedings/Association for the Advancement of Automotive Medicine. Association for the Advancement of Automotive Medicine, vol. 51, p. 485, 2007.

[4] E. Krakiwsky, C. Harris, and R. Wong, "A Kalman Filter for Integrating Dead Reckoning, Map Matching and GPS Positioning," in Proc. of IEEE Position Location and Navigation Symposium (PLANS'88). IEEE, 1988, pp. 39-46.

[5] B. Bradai, "Optimisation des Lois de Commande d'Éclairage Automobile par Fusion de Données," Ph.D. dissertation, Université de HauteAlsace, 2007.

[6] J. Lauffenburger, B. Bradai, M. Basset, and F. Nashashibi, "Navigation and Speed Signs Recognition Fusion for Enhanced Vehicle Location," in Proc. of International Federation of Automatic Control (IFAC'08), Seoul, Korea, 2008.

[7] A.-S. Puthon, F. Nashashibi, and B. Bradai, "Improvement of Multisensor Fusion in Speed Limit Determination by Quantifying Navigation Reliability," in Proc. of IEEE Intelligent Transportation Systems (ITSC'10). IEEE, 2010, pp. 855-860.

[8] "www.mobileye.com/menu/1/2/20/52/60."

[9] G. Piccioli, E. De Micheli, P. Parodi, and M. Campani, "Robust Method for Road Sign Detection and Recognition," Image and Vision Computing, vol. 14, no. 3, pp. 209-223, 1996.

[10] S. Maldonado-Bascon, S. Lafuente-Arroyo, P. Gil-Jimenez, H. GomezMoreno, and F. López-Ferreras, "Road-Sign Detection and Recognition based on Support Vector Machines," IEEE transactions on intelligent transportation systems, vol. 8, no. 2, pp. 264-278, 2007.

[11] A. Bargeton, F. Moutarde, F. Nashashibi, and B. Bradai, "Improving Pan-European Speed-Limit Signs Recognition with a New "Global Number Segmentation" Before Digit Recognition," in Proc. of IEEE Intelligent Vehicles Symposium (IV'08), Eindhoven, Netherlands, 2008, pp. 1022-1027.

[12] O. Hamdoun, A. Bargeton, F. Moutarde, B. Bradai, L. Chanussot et al., "Detection and Recognition of End-of-Speed-Limit and Supplementary Signs for Improved European Speed Limit Support," in Proc. of World Congress on Intelligent Transport Systems (ITS'08), vol. 15, New York, USA, 2008, pp. 289-303.

[13] C. Keller, C. Sprunk, C. Bahlmann, J. Giebel, and G. Baratoff, "RealTime Recognition of US Speed Signs," in Proc. of IEEE Intelligent Vehicles Symposium (IV'08), 2008, pp. 518-523.

[14] W. Wu, X. Chen, and J. Yang, "Detection of Text on Road Signs from Video," Intelligent Transportation Systems, vol. 6, no. 4, pp. 378-390, 2005.

[15] C. Bahlmann, M. Pellkofer, J. Giebel, and G. Baratoff, "Multi-Modal Speed Limit Assistants: Combining Camera and GPS Maps," in Proc. of IEEE Intelligent Vehicles Symposium (IV'08), 2008, pp. 132-137.

[16] D. Nienhuser, T. Gumpp, and J. Zollner, "A Situation Context Aware Dempster-Shafer Fusion of Digital Maps and a Road Sign Recognition System," in Proc. of IEEE Intelligent Vehicles Symposium (IV'09), Xi'an, Shaanxi, China, 2009, pp. 1401-1406.

[17] D. Mercier, B. Quost, and T. Denœux, "Contextual Discounting of Belief Functions," Symbolic and Quantitative Approaches to Reasoning with Uncertainty, pp. 552-562, 2005.

[18] A. Dempster, "Upper and Lower Probabilities Induced by a Multivalued Mapping," Classic Works of the Dempster-Shafer Theory of Belief Functions, pp. 57-72, 1967.

[19] G. Shafer, A Mathematical Theory of Evidence. Princeton university press Princeton, NJ, 1976.

[20] N. Hautière, J. Tarel, J. Lavenant, and D. Aubert, "Automatic Fog Detection and Estimation of Visibility Distance Through Use of an Onboard Camera," Machine Vision and Applications, vol. 17, no. 1, pp. 8-20, 2006.

[21] M. Roser and A. Geiger, "Video-based Raindrop Detection for Improved Image Registration," in Computer Vision Workshops (ICCV Workshops). IEEE, 2009, pp. 570-577.

[22] F. Nashashibi, R. De Charette De La Contrie, and A. Lia, "Detection of Unfocused Raindrops on a Windscreen using Low Level Image Processing," in Proc. of International Conference on Control Automation Robotics and Vision (ICARCV'10), Singapore, 2010. 